\title{
Nota crítica
}

\section{La noción de "fenómeno" en la fenomenología de Jean-Luc Marion}

\section{[The Notion of "Phenomenon" in Jean-Luc Marion's Phenomenology]}

\author{
JORGE LUIS ROGGERO \\ Consejo Nacional de Investigaciones Científicas y Técnicas \\ Universidad de Buenos Aires \\ jorgeluisroggero@gmail.com
}

\begin{abstract}
Resumen: Me propongo aclarar la noción de "fenómeno" de Jean-Luc Marion y su relación con la categoría de "fenómeno saturado". Para ello discutiré las interpretaciones de algunos comentaristas que advierten tensiones entre la primera y la segunda tópica del fenómeno.

Palabras clave: fenómeno saturado, tópica del fenómeno, automostración, grados de fenomenicidad, acontecialidad
\end{abstract}

\begin{abstract}
I aim to clarify Jean-Luc Marion's notion of "phenomenon" and its relationship with the category of "saturated phenomenon". To this end I discuss the interpretations of some commentators who point out tensions between the first and the second topic of the phenomenon presented.

Key words: saturated phenomenon, topic of the phenomenon, automonstration, degrees of phenomenality, eventuality
\end{abstract}

La fenomenología de la donación concede la iniciativa al fenómeno. En Étant donné, publicado originalmente en 1997, Jean-Luc Marion introduce el principio de la donación por el que se "renuncia a fundar el fenómeno para dejarle - por fin — la iniciativa de su aparición a partir de sí [soi]" (Marion 1998, p. 30; a menos que se indique lo contrario, las traducciones son mías). Marion asigna un soi al fenómeno a partir del cual el fenómeno aparece. Ciertamente, como él mismo admite (cfr. Marion 1998, p. 439), esto implica aceptar la noción heideggeriana de fenómeno como "lo-que-se-muestra-en-sí-mismo" (das Sich-an-ihmselbst-zeigende) (Heidegger 1977, p. 38). Sin embargo, el fenomenólogo francés hace algo más que sólo adoptar la concepción de fenómeno que presenta Heidegger en el § 7 de Sein und Zeit. Su propuesta implica una "radicalización", una exploración y un despliegue de las consecuencias más extremas de la definición heideggeriana. ${ }^{1}$

\footnotetext{
${ }^{1}$ Este aspecto ha sido bien advertido por Claudia Serban (cfr. Serban 2012, p. 84).
}

Revista de Filosofía Diánoia, vol. 65, no. 84 (mayo-octubre de 2020): pp. 167-189

e-ISSN: 1870-4913 • DOI: https://doi.org/10.22201/iifs.18704913e.2020.84.1586 
Ahora bien, en esta misma obra de 1997 Marion presenta su noción de "fenómeno saturado" (phénomène saturé) ${ }^{2}$ como el fenómeno que se da al contradecir toda condición de posibilidad impuesta a priori, e introduce una tópica del fenómeno en la que se distinguen tres tipos de fenómenos según su grado de donación: 1) los "fenómenos pobres en intuición" (phénomènes pauvres en intuition), 2) los "fenómenos de derecho común" (phénomènes de droit commun) y, por último, 3) los fenómenos saturados. Mientras que los fenómenos saturados parecen adaptarse bien a la idea heideggeriana de una automostración, los fenómenos pobres y los fenómenos de derecho común responden a una concepción del fenómeno constituido como objeto por las operaciones de un sujeto trascendental. En este sentido, cabe preguntarse si es posible aplicar la misma definición de "fenómeno" a todos los casos descritos por Marion.

A esta cuestión se suma la afirmación del fenómeno saturado como el fenómeno paradigmático. En el § 24 de Étant donné, Marion destaca que su proyecto es pensar el fenómeno pobre y el fenómeno de derecho común a partir del paradigma del fenómeno saturado ( $c f r$. Marion 1998, p. 316). Esta prioridad, entendida como una primacía de lo extraordinario sobre lo ordinario o de lo excepcional sobre lo normal, ha sido recusada por diversos comentaristas (entre otros, Anthony Steinbock, Emmanuel Falque y Christina Gschwandtner). Si el fenómeno saturado es el único capaz de adaptarse a la definición de fenómeno que ofrece Marion, entonces esta definición no parece capaz de dar cuenta de la totalidad de los fenómenos y, lo que sería más grave aún, no podría —según estos críticos- explicar los fenómenos más cotidianos.

En la conferencia "Saturation and Counter-Experience", presentada en el Congreso In Excess: Jean-Luc Marion and the Horizon of Modern Theology en la Universidad de Notre Dame en 2004, Marion responde a algunas de las críticas mediante la noción de "banalidad de la saturación". El texto en su versión definitiva se publicó como el capítulo titulado "La banalité de la saturation" del libro Le visible et le révélé (Marion 2005). La idea de la banalidad de los fenómenos saturados como respuesta a la crítica a su carácter extraordinario conduce a reducir la tópica del fenómeno a dos modos de fenomenicidad (cfr. Marion 2005, pp. 155-157). La nueva tópica del fenómeno, adoptada de manera definitiva en Certitudes négatives (cfr. Marion 2010, p. 301, n. 1)

\footnotetext{
${ }^{2}$ En rigor, esta categoría se presenta por primera vez en el texto "Le phénomène saturé" de 1992, pero es en Étant donné donde se terminan de definir sus características principales (cfr. Marion 1992).
}

Revista de Filosofía Diánoia, vol. 65, no. 84 (mayo-octubre de 2020) e-ISSN: 1870-4913 • DOI: https://doi.org/10.22201/iifs.18704913e.2020.84.1586 
y Reprise du donné (cfr. Marion 2016, pp. 147-189) distingue sólo dos tipos de fenómenos: los objetos y los acontecimientos.

En Degrees of Givenness, Gschwandtner señala cierto empobrecimiento de la fenomenicidad en la reducción de la posibilidad de gradación a sólo dos modos de manifestación. En un análisis de este cambio de clasificación, Vinolo (2017) sostiene que existe una tensión entre la metafísica y la hermenéutica en la obra de Marion que se plasma en una supuesta indecisión entre la primera y la segunda tópica del fenómeno.

Con el objeto de esclarecer la noción marioniana de "fenómeno", este artículo propone, en primer lugar, que no existe ninguna contraposición entre las dos tópicas del fenómeno; por el contrario, ambas clasificaciones se complementan. La nueva tópica — que reduce los fenómenos a dos tipos- puede articularse con la idea de una gradación de la fenomenicidad, pero además permite devolver el carácter crítico a la propuesta filosófica de Marion. ${ }^{3}$ Asimismo, y en segundo lugar, la nueva tópica permite comprender de modo acabado el estatus último del fenómeno en la obra marioniana y su relación con la prioridad que se otorga al fenómeno saturado.

En la primera sección me detendré en la lectura de Marion de la noción husserliana de fenómeno. En la segunda sección analizaré la primera tópica del fenómeno en la que Marion introduce su idea de "fenómeno saturado". En la tercera sección examinaré algunas objeciones y la respuesta que el filósofo francés ofrece a través de la segunda tópica del fenómeno. Por último, extraeré algunas conclusiones.

\section{La noción husserliana de "fenómeno"}

En Réduction et donation, Marion examina la crítica de Heidegger al "principio de todos los principios" en "Das Ende der Philosophie und die Aufgabe des Denkens":

La llamada "a la cosa misma", tanto en su sentido positivo como en el negativo, sirve para garantizar y elaborar el método; sirve de procedimiento filosófico, el único con el que la cosa misma llega a darse legítimamente. Para Husserl, el "principio de todos los principios" no es, en primer lugar, un principio de contenido, sino metodológico. [...] El "principio de todos los principios" implica la tesis de la primacía del método. Este principio

${ }^{3}$ Como bien destaca Jacob Rogozinski, el carácter crítico de la propuesta de Marion podía percibirse en la oposición entre icono e ídolo en sus obras teológicas tempranas (cfr. Rogozinski 2015, pp. 74-75).

Revista de Filosofía Diánoia, vol. 65, no. 84 (mayo-octubre de 2020) e-ISSN: 1870-4913 • DOI: https://doi.org/10.22201/iifs.18704913e.2020.84.1586 
decide sobre cuál es la única "cosa" que pueda convenirle al método. Exige que la subjetividad absoluta sea la "cosa" de la Filosofía. [...] Si se preguntara ¿de dónde saca el "principio de todos los principios" su inamovible legitimidad?, habría entonces que responder: de la subjetividad, que se ha dado ya por supuesto es la "cosa" de la Filosofía. (Heidegger 2007, pp. 77-78)

Según Heidegger, Husserl contradice la máxima fenomenológica del "retorno a las cosas mismas" al formular el "principio de todos los principios", ya que éste conduce, más que a las cosas mismas, a un retorno a la evidencia que la intuición otorga a la conciencia. Según Marion, lo que la crítica de Heidegger señala es el desplazamiento desde una ciencia de los fenómenos hasta una "ciencia de y por la conciencia" (Marion 1989, p. 80).

Sin embargo, si bien Marion comparte la crítica de Heidegger, el fenomenólogo francés destaca que el principio husserliano también pretende asegurar la primacía del fenómeno al enfatizar que éste debe considerarse "como se da" (als was es sich gibt). Es fundamental advertir que aquí se encuentra en germen la concepción heideggeriana de fenómeno como "lo-que-se-muestra-en-sí-mismo". No obstante, también es importante entender que esta donación del fenómeno como se da se encuentra restringida por la conciencia misma. El "principio de todos los principios" - señala Marion- interpreta la donación como donación de una presencia efectiva para la conciencia y en vistas a la certeza ( $c f r$. Marion 1989, pp. 81-82).

Según Marion, la fenomenicidad y el fenómeno adquieren los siguientes rasgos en la concepción husserliana:

1) En primer lugar, la conciencia determina de antemano el modo de ser de los fenómenos: la objetividad, único modo que permite la presencia efectiva y cierta del fenómeno (cfr. Marion 1989, pp. 82-83).

2) En segundo lugar, Marion observa que lo que aparece no se admite simplemente porque aparece, sino porque aparece en una instancia originaria. La legitimidad de lo que aparece se da por la instancia originaria de la intuición. Marion se pregunta cómo hay que entender la noción de una gebende Anschauung (Husserl 1976, p. 51) o una gebende Intuition (Husserl 1976, p. 46) y constata que es la intuición la que da y nunca la aparición. En este 
sentido, la intuición tiene la última palabra, pues impone sus condiciones al aparecer (cfr. Marion 1989, p. 83). ${ }^{4}$

3) Por último, Marion destaca que la indicación más clara de la sumisión de la fenomenicidad a la conciencia se da por la dualidad que se origina por la definición del fenómeno que se despliega a partir de la vivencia. ${ }^{5}$ Existe una dualidad irreductible en la noción husserliana de fenómeno entre un polo subjetivo (das Erscheinen) y otro objetivo (das Erscheinendes). "Fenómeno" designa tanto "el aparecer" como "lo que aparece". Marion observa que esta dualidad, que se modula en diversos pares (intención/intuición, significación/cumplimiento, nóesis/nóema), lleva a Husserl a postular que la manifestación más plena de un fenómeno sucede cuando hay una adecuación perfecta entre ambos aspectos, cuando el aparecer subjetivo equivale al aparecer objetivo (cfr. Marion 1998, pp. 265-266). Lo problemático de este planteamiento — según observa Marion- es que esa adecuación perfecta no es la norma, sino la excepción. ${ }^{6}$ En este sentido, la intuición husserliana es una intuición deficiente, una intuición pobre. La adecuación sólo se alcanza en los ámbitos en los que la intención no reclama más que una intuición formal (por ejemplo, el espacio en matemáticas), una intuición categorial (por ejemplo, las entidades lógicas)

${ }^{4}$ De este modo, Marion se hace eco de la célebre observación de Paul Ricœur al "principio de todos los principios" en su traducción de Ideen I. Ricœur encuentra llamativo el contraste entre una "intuición donadora" (intuition donatrice) y "lo que se da" (ce qui se donne) y sostiene que "resume de manera abreviada todas las dificultades de la filosofía de la constitución que debe ser al mismo tiempo, desde otro punto de vista, un intuicionismo" (Ricœur 1950, p. 78, n. 1). En Étant donné, Marion menciona la observación de Ricœur (cfr. Marion 1998, p. 21, n. 1 y p. 98, n. 2).

${ }^{5}$ El propio Husserl señala la equivocidad de la noción de fenómeno en el $\S 2$ de su quinta investigación lógica: "No será señalado nunca con bastante rigor el equívoco que permite llamar fenómeno [Erscheinung], no sólo a la vivencia en que consiste el aparecer [das Erscheinen] del objeto (por ejemplo, la vivencia concreta de la percepción en que el objeto mismo no está supuestamente presente), sino también al objeto que aparece [das erscheinende Objekt] como tal" (Husserl 1984, p. 359). En Die Idee der Phänomenologie, Husserl afirma: "La palabra 'fenómeno' [Phänomen] es ambigua por la correlación esencial entre aparecer [Erscheinen] y lo que aparece [Erscheinendes]. Phainómenon significa propiamente 'lo que aparece' y, sin embargo, se utiliza con frecuencia para designar el aparecer mismo, el fenómeno subjetivo (si se permite usar esta expresión que puede inducir a malentendidos si se la toma en un sentido psicológico tosco)" (Husserl 1984, p. 14).

${ }^{6}$ En la sexta investigación lógica Marion se detiene en las diversas figuras de la verdad como adecuación enunciadas por Husserl (cfr. Husserl 1984, pp. 645-656).

Revista de Filosofía Diánoia, vol. 65, no. 84 (mayo-octubre de 2020) e-ISSN: 1870-4913 • DOI: https://doi.org/10.22201/iifs.18704913e.2020.84.1586 
o incluso ninguna intuición (por ejemplo, la tautología vacía) (cfr. Marion 1998, p. 268).

Según Marion, esta penuria de la intuición es el resultado de un supuesto metafísico que no se cuestiona. Husserl sigue a Kant en su definición de la verdad como adaequatio y el paralelismo entre intuición y concepto que ésta implica. Pero, ¿es esto así? ¿Puede decirse sin más que Husserl sigue a Kant en estas cuestiones? ${ }^{7}$ Sin duda, no cabe reducir la propuesta husserliana a la kantiana. Es cierto que el concepto husserliano de fenómeno es más amplio que el kantiano ${ }^{8}$ y que no puede pensarse la fenomenología trascendental como un mero regreso al idealismo trascendental kantiano; no obstante, considero que el problema de estas críticas es que no advierten la estrategia de lectura cuasi-deconstructiva de Marion y que siempre presenta un doble movimiento. Marion no reduce Husserl a Kant ni tampoco a algunos de sus textos. Por el contrario, su objetivo es siempre demostrar que la propuesta de la fenomenología de la donación ya se encontraba como una posibilidad latente en la fenomenología husserliana. Sin embargo, para establecer la importancia del desarrollo de esas posibilidades, es necesario dar cuenta de aquello que en la propia obra de Husserl impidió ese desarrollo. En palabras del propio Marion: "a menudo, Husserl no ve lo que abre como posibilidad, mientras que lo que cree ver mejor, en ocasiones, cierra la posibilidad" (Marion 1989, p. 247). En este caso, se trata de: 1) mostrar cómo la noción de objetividad —que ciertamente tiene raigambre kantiana- ${ }^{9}$ dejó su huella en el concepto de fenómeno que Husserl presentó en su obra publicada, es decir, en la obra que el propio autor eligió para difundir su pensamiento; 2) explorar la lógica de la objetividad hasta sus últimas consecuencias. En este sentido, el desplazamiento de la problemática al propio Kant es válido, pues

\footnotetext{
${ }^{7}$ Eduardo González di Pierro señala que éste es precisamente el error de Marion (cfr. González di Pierro 2011, p. 126).

${ }^{8}$ Basta mencionar la amplitud con la que se presenta la noción de fenómeno en la lección inaugural de 1917. Allí Husserl define el fenómeno como lo que se refiere a todos los modos de ser consciente de algo, incluido "todo tipo de sentimiento, deseo y querer con su comportamiento inmanente" (Husserl 1987, p. 71).

${ }^{9}$ Sostiene Marion en La rigueur des choses: "Husserl, al pensar o al formular en forma espontánea todo fenómeno en el horizonte de la objetividad reproduce el gesto de Kant, para quien va de suyo que los fenómenos son un caso particular del objeto, ya que el objeto es el concepto más elevado — como lo proclama y postula la conclusión de la 'Analítica' de la primera Crítica-, el concepto englobante de todo fenómeno posible, incluso de nosotros mismos" (Marion 2012a, p. 127).
}

Revista de Filosofía Diánoia, vol. 65, no. 84 (mayo-octubre de 2020) e-ISSN: 1870-4913 • DOI: https://doi.org/10.22201/iifs.18704913e.2020.84.1586 
se trata de establecer con claridad la naturaleza del obstáculo que la fenomenología de la donación pretende superar.

Como bien destaca Jean-François Lavigne, es el propio Husserl quien define a su proyecto filosófico como un "idealismo trascendental" (§ 41 de Cartesianische Meditationen) y, si bien esto conlleva una nueva acepción de estos términos, cabe indagar el motivo por el que Husserl retoma este sintagma (Lavigne 2003, p. 18).

Lavigne señala que esta elección husserliana implica que su fenomenología efectúa la tendencia propia del idealismo kantiano pero, al radicalizarla, supera sus límites y contradicciones. ¿En qué sentido? Por "idealismo trascendental" — según explica con claridad Lavignese entiende la tesis según la cual los objetos de nuestro conocimiento tienen sólo una realidad empírica, es decir, sólo poseen una existencia efectiva cuando se refieren a una posible experiencia vivida. Los objetos son en la medida en que se nos aparecen. Pero, además, estos objetos son ideales desde el punto de vista trascendental, pues deben toda su realidad a operaciones del pensamiento. La tesis gnoseológica del idealismo crítico supone también una afirmación ontológica. Tal es el concepto de "idealismo trascendental" (kantiano y fichteano) que hereda Husserl. El § 41 de Cartesianische Meditationen confirma el tipo de continuidad: la diferencia entre el "idealismo trascendental" clásico y el husserliano no radica en el contenido de sus posiciones metafísicas, sino en el alcance que este último le da a la fundación idealista de la objetividad por parte de la actividad sintética subjetiva (cfr. Lavigne 2003, p. 20):

Llevada a cabo en esta concreción sistemática, la fenomenología es eo ipso "idealismo trascendental", bien que en un sentido radicalmente nuevo. No es en el de un idealismo psicológico, en el de un idealismo que se empeña en sacar de datos sensoriales sin ningún sentido un mundo con un sentido. Tampoco es un idealismo kantiano, que cree poder dejar abierta, al menos como concepto límite, la posibilidad de un mundo de cosas en sí; sino un idealismo que, exactamente, no es nada más que una autoexposición de mi ego en cuanto sujeto de todo conocimiento posible. (Husserl 1973, p. 118)

En este sentido, el idealismo husserliano se distingue del kantiano en que no permite ningún "mundo de cosas en sí", sino que toda posible dimensión ontológica queda absorbida en el campo de la vida intencional. Comparto, pues, la conclusión de Lavigne: "la fenomenología husserliana no es idealista y trascendental de un modo distinto

Revista de Filosofía Diánoia, vol. 65, no. 84 (mayo-octubre de 2020) e-ISSN: 1870-4913 • DOI: https://doi.org/10.22201/iifs.18704913e.2020.84.1586 
al que lo era el criticismo; sólo lo es de modo más radical" (Lavigne 2003, p. 21). ${ }^{10}$

Sin duda, esto no implica que no existan otras posibilidades en la propuesta fenomenológica de Husserl que pueden explorarse en un sentido distinto al del "idealismo trascendental", y que no puedan rastrearse lineamientos en este sentido en muchas de sus obras; no obstante, Lavigne acierta al destacar la relevancia que el propio Husserl otorga a la comprensión de su obra como un "idealismo trascendental".

Así, Marion analiza la lógica kantiana de la objetividad que habita este "idealismo trascendental" y que se juega en el equilibrio entre intuición y concepto. Kant es claro respecto del funcionamiento de cada facultad:

Sin sensibilidad no nos sería dado objeto alguno; y sin entendimiento, ninguno sería pensado. Pensamientos sin contenidos son vacíos, intuiciones sin conceptos son ciegas. Por eso, es tan necesario hacer sensibles sus conceptos (es decir, añadirles el objeto en la intuición) como hacer inteligibles sus intuiciones (es decir, llevarlas bajo conceptos). Tampoco pueden estas dos facultades, o capacidades, trocar sus funciones. El entendimiento no puede intuir nada, y los sentidos no pueden pensar nada. (Kant 19031904)

El problema es que la equivalencia entre intuición y concepto no es tal. Según Marion, Kant no termina de advertir la prioridad de la intuición sobre el concepto porque, si bien el concepto es vacío sin intuición, la intuición es simplemente ciega sin concepto, pero todavía da (cfr. Marion 1998, p. 270).

En este sentido, no sólo no se traza un paralelo entre la intuición y el concepto, sino que la intuición asegura la posibilidad del concepto. Sin embargo, en lugar de subrayar este privilegio, Marion afirma que Kant estigmatiza la deficiencia de la intuición circunscribiéndola a los límites de la sensibilidad finita (cfr. Marion 1998, pp. 271-272).

\section{La tópica del fenómeno}

A partir de la constatación del privilegio histórico que se le ha dado a los fenómenos lógicos y matemáticos como modelos de certeza, es

${ }^{10}$ Dominique Pradelle también comparte esta opinión: "la fundación de una ciencia de la dimensión trascendental no podría significar una refutación de la revolución copernicana de Kant, sino que, por el contrario, constituye su radicalización" (Pradelle 2012, p. 352). 
decir, a los fenómenos que se dan bajo el "régimen de la deficiencia de intuición", Marion concluye que este paradigma de la pobreza de intuición: 1) excluye el acceso a la mayoría de los "fenómenos corrientes" y 2) no contempla la posibilidad contraria: la existencia de fenómenos que se den con un exceso de intuición (cfr. Marion 1998, pp. 273-280). En su opinión, dentro de las posibilidades que ofrece la dualidad misma del fenómeno está la de un fenómeno que recibiría una demasía de la intuición respecto de la intención, el concepto o la mención. Este fenómeno saturado de intuición no podría ser objetivado, pero debe considerarse ya que constituye una posibilidad de la fenomenicidad. "Y, en fenomenología, la más mínima posibilidad nos obliga" (Marion 1998, p. 280).

Por lo tanto, se trata de desplegar una posibilidad de la fenomenología o, si se prefiere, de desplegar la posibilidad de la posibilidad, de liberarla de su subordinación al "poder de conocer", otorgando el "poder de aparecer" al fenómeno mismo. ${ }^{11}$

En razón de estas consideraciones, y con la adopción expresa de la definición heideggeriana de fenómeno, ${ }^{12}$ Marion propone una tópica de los diferentes tipos de fenómenos posibles: 1) fenómenos pobres en intuición, 2) fenómenos de derecho común y 3) fenómenos saturados.

1) Los fenómenos pobres son los fenómenos que requieren sólo una intuición formal (matemáticas) o una intuición categorial (lógica). A estos fenómenos les basta con la mera inteligibilidad de su concepto para darse. Éstos son los casos que, gracias a su déficit de intuición, la metafísica convierte en paradigmas de certeza epistémica abstracta (cfr. Marion 1998, pp. 310-311).

2) Los fenómenos de derecho común son fenómenos que se manifiestan en cuanto que obtienen cierto cumplimiento intuitivo. Pero

${ }^{11}$ Según Marion, "Las condiciones formales del conocimiento se articulan aquí de manera directa a partir del poder de conocer y no a partir del poder de aparecer del fenómeno. Esto significa que la intuición y el concepto determinan por adelantado la posibilidad de aparecer de todo fenómeno. La posibilidad — como también, y sobre todo, la imposibilidad- de un fenómeno se decreta a la medida del 'poder de conocer', del juego de la intuición y del concepto en un espíritu finito. Por lo tanto, todo fenómeno que concuerde con la finitud del poder de conocer y con sus exigencias es posible" (Marion 1998, p. 254).

12 "Mantendremos de entrada en todos los casos la definición genérica como lo que se muestra en y a partir de sí (Heidegger), lográndolo sólo porque se da en sí y a partir solamente de sí [...]. Los diferentes tipos de fenómenos pueden definirse como variaciones de la automanifestación (mostrarse en y a partir de sí) siguiendo el grado de donación (darse en y a partir de sí)" (Marion 1998, pp. 309-310).

Revista de Filosofía Diánoia, vol. 65, no. 84 (mayo-octubre de 2020) e-ISSN: 1870-4913 • DOI: https://doi.org/10.22201/iifs.18704913e.2020.84.1586 
basta con una confirmación intuitiva débil del concepto para dar el fenómeno correspondiente si éste se confirma repitiéndose con regularidad. Entre los fenómenos de derecho común se encuentran los objetos de la física y de las ciencias naturales. Este tipo de fenómenos que proceden por objetivación requieren, según Marion, que lo dado intuitivamente sea restringido a lo que permite confirmar el concepto (cfr. Marion 1998, p. 312).

El caso paradigmático de fenómeno de derecho común es el "objeto técnico" (objet technique). En estos casos, el concepto y la intención son el "plano", "esquema" o "diseño" que permite un "mostrar por concepto" (montrer par concept) (Marion 1998, p. 312). De este modo, los objetos técnicos se caracterizan por el retraso y la previsión. El retraso está dado por la manifestación por "concepto" que precede a su donación. La producción del objeto técnico sólo completa "anecdóticamente" al concepto, pues el producto no se encuentra dado en primer lugar, sino siempre $a$ posteriori y esa donación ya se encuentra prevista por entero en las exigencias del concepto (cfr. Marion 1998, p. 313).

3) Por último, los fenómenos saturados o "paradojas" son fenómenos en los que la intuición desborda la expectativa de la intención, que aparecen cuando se despejan las dos condiciones que Husserl impone a la mostración de los fenómenos (el horizonte y el Yo). Mediante la presentación de lo que podría considerarse —según la expresión de Falque- una "suerte de Crítica de la razón pura invertida" (Falque 2014, p. 138), Marion aborda la idea de saturación a partir de una inversión de las categorías kantianas. El fenómeno saturado excede las categorías porque en él la intuición sobrepasa todo concepto.

\section{En este sentido:}

1) En primer lugar, el fenómeno saturado es no-mentable (invisable) según la cantidad, pues es imprevisible. El fenómeno saturado es un fenómeno inconmensurable porque en él no es aplicable ninguna "síntesis sucesiva", sino que más bien habría que intentar una "síntesis instantánea" que sobrepasa la síntesis de sus componentes eventuales y no puede ser prevista (cfr. Marion 1998, p. 281).

2) En segundo lugar, el fenómeno saturado es insoportable (insupportable) según la cualidad. A partir de cierto grado, la intensidad 
de la intuición supera todas las anticipaciones conceptuales de la percepción. El fenómeno saturado contempla la posibilidad de un exceso en la intuición de tal magnitud que la mirada experimenta un "deslumbramiento" (éblouissement) en el que la percepción ya no puede anticipar lo que va a recibir de intuición (cfr. Marion 1998, p. 285).

3) En tercer lugar, el fenómeno saturado es absoluto (absolu) según la relación, es decir, se sustrae a toda posible analogía de la experiencia. Sin embargo, esto no implica que el fenómeno pueda dispensarse de un horizonte en general, puesto que ello impediría toda manifestación: "se trata de usar el horizonte de otro modo para liberarse de su anterioridad delimitadora" (Marion 1998, p. 293). ${ }^{13}$

4) En cuarto lugar, el fenómeno saturado es "inmirable" (irregardable) según la modalidad. Esta última categoría se asocia a la acepción trascendental del Yo. Como bien destaca Marion, lo que está en juego es la concordancia de los objetos con el "poder de conocer" (cfr. Marion 1998, p. 297). En palabras de Kant: "El postulado de la posibilidad de las cosas exige que el concepto de ellas concuerde con las condiciones formales de una experiencia en general" (Kant 1903-1904, A 220/B 267). Sin embargo, de este modo - señala Marion-, al someter "el poder de aparecer" al "poder de conocer", se priva de "autonomía fenoménica" al fenómeno, se provoca la alienación del fenómeno respecto de sí mismo. Marion propone invertir el dispositivo kantiano para preguntar: "¿qué sucedería si un fenómeno no 'concordara' y no 'correspondiera' con el poder de conocer del Yo?” (Marion 1998,

${ }^{13}$ A partir de esta consideración, Marion propone tres figuras de la saturación: la primera figura se cumple en el horizonte, pero contra él. La intuición alcanza los límites del concepto y del horizonte, pero no los sobrepasa. El fenómeno colma el horizonte, produce un deslumbramiento y se retira (cfr. Marion 1998, p. 293). La segunda figura se da cuando el fenómeno saturado de intuición colma y sobrepasa la delimitación del horizonte. En este caso es necesario articular varios horizontes de modo conjunto para recibir al fenómeno (cfr. Marion 1998, p. 294). Marion propone, pues, que es necesario aceptar fenómenos con $n+1$ horizontes. En estos casos, el deslumbramiento da lugar a una hermenéutica infinita (cfr. Marion 1998, p. 295). La tercera figura surge al considerar la posibilidad de que, en lugar de combinarse, los múltiples horizontes sumen sus deslumbramientos. En este caso —que es el del fenómeno de la revelación- se daría un redoblamiento de la saturación que ningún horizonte ni ninguna combinación de horizontes sería capaz de tolerar (cfr. Marion 1998, p. 295).

Revista de Filosofía Diánoia, vol. 65, no. 84 (mayo-octubre de 2020) e-ISSN: 1870-4913 • DOI: https://doi.org/10.22201/iifs.18704913e.2020.84.1586 
p. 298). En términos kantianos, ese fenómeno no aparecería. No obstante, reflexiona Marion, la incapacidad del Yo para objetivar no debería funcionar como una limitación respecto de la aparición del fenómeno. Marion distingue entre "mirar" (regarder) y "ver" (voir). El fenómeno saturado se deja ver, pero no mirar, pues "mirar" implica mantener (garder) lo visible bajo el control del que mira, mientras que "ver" es más bien "recibir lo que se muestra desde sí" (Marion 1998, p. 299).

Ahora bien, si el fenómeno saturado aparece en contradicción con toda condición de posibilidad de la experiencia, ¿̇cómo es posible su experiencia? Marion responde que debe pensarse en una experiencia no objetiva que puede entenderse como "contraexperiencia" (contre-expérience), como una experiencia que consiste justo en ser una "experiencia de lo que contradice en forma irreductible las condiciones de la experiencia de los objetos" (Marion 1998, pp. 300-301).

Frente al fenómeno saturado, el Yo puede verlo, pero ya no fijarlo o controlarlo. Es más, el fenómeno saturado invierte la relación intencional con el Yo. No sólo el Yo ya no puede constituir al fenómeno, sino que es constituido por él. El sujeto constituyente deviene un testigo constituido (témoin constitué) (cfr. Marion 1998, p. 302).

\section{La nueva tópica del fenómeno}

En "Phénoménologie de l'extraordinaire", Emmanuel Falque se pregunta por la pertinencia de concebir el fenómeno saturado como fenómeno paradigmático. ¿Cuáles son las consecuencias de hacer de la excepción la norma? Falque analiza el siguiente pasaje del § 23 de Étant donné:

Todo nuestro proyecto [...] intenta pensar el fenómeno de derecho común y, a través de él, el fenómeno pobre a partir del paradigma del fenómeno saturado; uno y otro ofrecen así variantes debilitadas y derivadas de este último, como si se tratara de extenuaciones progresivas del fenómeno saturado que no se da al margen de la norma ni como una excepción a la definición de la fenomenicidad; al contrario, corresponde propiamente al fenómeno saturado convertir en pensable la medida de la manifestación a partir de la donación y reencontrarla hasta en su variación de derecho común, incluso en el fenómeno pobre. Lo que la metafísica descarta como una excepción (la paradoja saturada), la fenomenología lo adopta como su norma: todo fenómeno se muestra en la medida (o la desmedida) en que se da. (Marion 1998, p. 316) 
Si la excepción deviene la norma, si lo extraordinario se vuelve el paradigma para juzgar lo ordinario ¿qué ocurre con la cotidianeidad del hombre que, inmediata y regularmente, se ve sometida a la penuria de intuición más que a la saturación ${ }^{14}$ Establecer un paradigma basado en la experiencia extraordinaria de la saturación no es una decisión acertada —según Falque- porque no ayuda a comprender la experiencia más común del hombre, la experiencia ordinaria de la finitud humana. ${ }^{15}$

En "La banalité de la saturation" encontramos la respuesta de Marion a esta crítica. ${ }^{16}$ Allí Marion explica que existe un "motivo serio" que hace que aun hoy persista la reticencia frente a la hipótesis del fenómeno saturado. Este motivo es el miedo a que se trate de fenómenos que se dan en "intuiciones excepcionales" (Marion 2005, p. 87) regidas por la modalidad "maximalista" — según la expresión de Janicaud(cfr. Janicaud 2009, p. 69), es decir, el miedo a que se trate de fenómenos que se alcanzan rara vez en una especie de éxtasis confuso y fuera de lo común.

Marion responde a esta objeción mediante la distinción entre frecuencia y banalidad. Sin duda, los fenómenos pobres y los fenómenos comunes son frecuentes, y esta "frecuencia" tiene una consecuencia decisiva:

Podemos incluso decir que el mundo está cubierto de una capa, invasiva, la más visible, de fenómenos pobres, de objetos técnicos producidos y reproducidos sin fin, que termina por ofuscar lo que recubre. ¿Y qué recubre sino otros fenómenos [...] que hemos propuesto llamar fenómenos saturados? (Marion 2005, pp. 154-155)

En este sentido, en el del "encubrimiento", los fenómenos saturados pueden pensarse como poco frecuentes. Pero esta poca frecuencia no impide que consideremos a estos fenómenos banales. La banalidad se diferencia de la frecuencia. "Deviene banal en sentido estricto lo que [...] concierne a todos y deviene accesible a todos" (Marion 2005,

14 “¿Qué queda de lo no excepcional o, al menos, de lo no 'excesivo' como tal que, sin embargo, hace a lo esencial de la 'finitud del adonado'?" (Falque 2014, p. 142).

${ }^{15}$ Falque señala que Marion cae en la "preferencia de lo infinito sobre lo finito", en la que cae también Lévinas y buena parte de la nouvelle phénoménologie (cfr. Falque 2004, pp. 40-44).

${ }^{16}$ El propio Falque reconoce que se trata de una respuesta a su objeción en su reescritura del artículo para su libro Le combat amoureux. "El ensayo 'La banalité de la saturation' podría ciertamente interpretarse como una respuesta a la objeción que se formula aquí" (Falque 2014, p. 143, n. 7).

Revista de Filosofía Diánoia, vol. 65, no. 84 (mayo-octubre de 2020) e-ISSN: 1870-4913 • DOI: https://doi.org/10.22201/iifs.18704913e.2020.84.1586 
p. 155). Este tipo de banalidad, disponible para todos, no es equivalente a la frecuencia e incluso puede oponerse a ella. Explica Marion:

Hablar de un fenómeno saturado banal no implica entonces que devenga corriente y frecuente; ni que, a contrario, devenga obligatoriamente excepcional y raro, y por lo tanto se restrinja a los márgenes de la fenomenicidad común que supuestamente fija la norma. La banalidad del fenómeno saturado sugiere, a la inversa, que la mayoría de los fenómenos, si no todos, pueden dar lugar a la saturación por exceso en ellos de intuición sobre el concepto o la significación. En otros términos, la mayoría de los fenómenos, que aparecen a primera vista como pobres en intuición, podrían describirse no sólo como objetos, sino también como fenómenos en los que, por lo tanto, la intuición satura y desborda todo concepto unívoco. Ante la mayoría de los fenómenos, incluso los más someros (la mayoría de los objetos producidos por la técnica y reproducidos de manera industrial), se abre la posibilidad de una doble interpretación que sólo depende de las exigencias de mi relación, siempre cambiante, con ellos. O, más bien, cuando la descripción lo exige, tengo a menudo la posibilidad de pasar de una interpretación a la otra, de una fenomenicidad pobre o común a una fenomenicidad saturada. (Marion 2005, pp. 155-156)

De este modo - al introducir la idea de la banalidad de los fenómenos saturados en respuesta a la acusación de su carácter extraordinario-, Marion reduce la tópica de los fenómenos a "dos modos de fenomenicidad" (Marion 2005, p. 157).

Estos dos modos de fenomenicidad recibirán el nombre de "objetos" y "acontecimientos". En su libro de 2010, Certitudes negatives, y en continuidad con la consideración hecha en "La banalité de la saturation", Marion propone sustituir la tópica del fenómeno presentada en el § 23 de Étant donné por una nueva clasificación:

De este modo, se dispone de una nueva tabla de fenómenos. Por un lado, los fenómenos del tipo del objeto, que comprenden los fenómenos pobres (formas lógicas, entidades matemáticas, etc.) y los fenómenos de derecho común (objetos de las ciencias de la "naturaleza", objetos industriales, etc.). Por el otro, los fenómenos del tipo del acontecimiento, que comprenden los fenómenos saturados simples (el acontecimiento en sentido estricto, según la cantidad; el ídolo o el cuadro, según la cualidad; la carne, según la relación; y el ícono o rostro del otro según la modalidad), pero también los fenómenos de revelación (que combinan diversos fenómenos saturados, como el fenómeno erótico, los fenómenos de revelación, la Revelación, etc.). Esta tabla completa y complica la de Étant donné [...] al ligar la saturación y la acontecialidad: un fenómeno se muestra tanto

Revista de Filosofía Diánoia, vol. 65, no. 84 (mayo-octubre de 2020)

e-ISSN: 1870-4913 • DOI: https://doi.org/10.22201/iifs.18704913e.2020.84.1586 
más saturado cuando se da con una acontecialidad más grande. (Marion 2010, p. 301, n. 1)

La nueva tabla de fenómenos reduce todos los fenómenos a dos tipos. De nuevo, como se sostuvo en "La banalité de la saturation", el paso de un tipo a otro puede realizarse por medio de una interpretación:

La distinción de modos de fenomenicidad (para nosotros entre el objeto y el acontecimiento) puede articularse sobre variaciones hermenéuticas que, como existenciarios del Dasein, tienen autoridad (ontológica) sobre la fenomenicidad de los entes. Sólo depende de mi mirada que incluso una piedra pueda aparecer, a veces, como un acontecimiento [... . . La distinción de los fenómenos en objetos y acontecimientos encuentra, por lo tanto, un fundamento en las variaciones de la intuición. Cuanto más aparece un fenómeno como acontecimiento (se acontecializa), más resulta saturado de intuición. Cuanto más aparece como objeto (se objetiviza), más resulta pobre de intuición. O, más aún: la acontecialidad fija el grado de la saturación y la saturación varía según la acontecialidad. Esta distinción tiene por ende un estatus estrictamente fenomenológico. Pero, entonces, hay que destacar asimismo que la acontecialidad no caracteriza sólo a uno de los tipos de fenómeno saturado (el acontecimiento stricto sensu, por oposición al ídolo, a la carne y al ícono): no sólo determina a cada uno de estos tipos que la ponen en práctica, sino que también define al fenómeno como dado en general, pues todos los fenómenos, en un grado o en otro, aparecen como advienen, ya que incluso los objetos técnicos no pueden borrar por completo los vestigios en ellos de un advenir, aun cuando esté oscurecido. (Marion 2010, pp. 307-308)

Una vez más, Marion no sólo reduce las posibilidades de la fenomenicidad a dos tipos, sino que introduce la posibilidad del paso de una a otra por medio de "variaciones hermenéuticas" determinadas por el tipo de mirada. ${ }^{17}$

Tras estas respuestas de Marion, la objeción de Falque es retomada de alguna manera primero por Anthony Steinbock en su artículo de

${ }^{17}$ Esta distinción entre objetos y acontecimientos como nueva tópica de los fenómenos se retoma en el reciente Reprise du donné (2016), en el capítulo final. Allí Marion critica con severidad el "primado del objeto" en la historia de la filosofía, que aliena a la cosa en sí. Pero también destaca cómo no dejamos de experimentar aquello que no se deja constituir como objeto. Más allá de la objetivación, todo fenómeno conserva la posibilidad de aparecer a partir de sí mismo. Este carácter acontecial se registra en la donación de todo tipo de fenómeno (cfr. Marion 2016, pp. 147-189). Vuelvo sobre esta cuestión en el cuerpo del texto.

Revista de Filosofía Diánoia, vol. 65, no. 84 (mayo-octubre de 2020) e-ISSN: 1870-4913 • DOI: https://doi.org/10.22201/iifs.18704913e.2020.84.1586 
2007, "The Poor Phenomenon", y luego por Christina Gschwandtner en su ya mencionado libro, Degrees of Saturation. Steinbock sostiene que, en la obra de Marion, el estatus del fenómeno pobre permanece ambiguo. Este estatus debe aclararse porque lo que está en juego son las experiencias cotidianas. ${ }^{18}$ Según Steinbock, Marion se equivoca al sostener que el fenómeno pobre debe determinarse con base en el fenómeno saturado, pues de este modo no parece posible sostener la irreductibilidad de las "tres figuras originarias de la fenomenicidad" (Marion 1998, p. 310). Si el fenómeno saturado es el fenómeno paradigmático, ¿qué estatus tienen los demás fenómenos? ${ }^{19}$ Por su parte, siguiendo a Steinbock, Gschwandtner sostiene que es necesario introducir grados de donación y de saturación. ${ }^{20}$

Además, según señala Stéphane Vinolo, la nueva tópica del fenómeno parece implicar un desplazamiento de una distinción metafísica cualitativa entre diferentes tipos de fenómenos a una distinción cuantitativa que depende de una hermenéutica que ejerce la mirada. ${ }^{21}$

18 “¿Es la pobreza del fenómeno pobre intrínseca a las cosas mismas? ¿Los fenómenos pobres se dan de modo uniforme? ¿Hay un déficit o una corrupción en la donación que se debe a nosotros, a nuestra inatención, o a nuestra inhabilidad para recibir fenómenos saturados? ¿Los fenómenos pobres son características esenciales o contingentes de nuestra existencia?" (Steinbock 2007, p. 358).

19 “¿Todo lo demás es, en última instancia, reductible al fenómeno saturado? [...] ¿Los otros fenómenos son modos derivados?" (Steinbock 2007, p. 360).

${ }^{20}$ Según Gschwandtner, resulta problemático considerar que todo fenómeno que no se da con una saturación extrema cae en la objetividad, pues esto no refleja nuestra experiencia cotidiana (cfr. Gschwandtner 2014, pp. 8-9).

${ }^{21}$ Dice Vinolo: "Por un lado, Marion establece entre los fenómenos rupturas cualitativas que le permiten hablar de tipos de fenómenos. Así podemos postular categorías fenoménicas con el objeto de distinguir entre los fenómenos pobres, los fenómenos de derecho común, los fenómenos saturados y los fenómenos de revelación. [...] Sin embargo, desde otra vertiente de su fenomenología, estas rupturas cualitativas dejan de manera repentina lugar a una simple continuidad cuantitativa que vemos que ocupa su lugar de modo progresivo en diferentes textos publicados entre 2001 y 2012. [...] Apartándose de la voluntad metafísica de descubrir categorías, Marion se acerca entonces a la hermenéutica. Este momento cuantitativo y continuista de la obra es identificable con claridad. Si bien aparece en varios textos, se cristaliza en la idea de 'banalidad' de la saturación (Marion, 2005b, pp. 143182), que reduce lo que presentaba como tipos a simples interpretaciones posibles de éstos. [...] Aquí la saturación no es más un tipo de fenómeno, sino una interpretación siempre ya posible de 'la mayoría' de ellos porque están relacionados cuantitativamente y ya no separados cualitativamente" (Vinolo 2017, pp. 344-345).

Revista de Filosofía Diánoia, vol. 65, no. 84 (mayo-octubre de 2020) e-ISSN: 1870-4913 • DOI: https://doi.org/10.22201/iifs.18704913e.2020.84.1586 


\section{Todo fenómeno es un fenómeno saturado}

Estas objeciones señalan sin duda una cuestión fundamental en la obra de Marion. ¿Cómo hay que entender el paradigma del fenómeno saturado? ¿Es el fenómeno saturado uno de los tres tipos de fenómenos o es el fenómeno originario del cual los otros dos se derivan? La "nueva tópica de los fenómenos", que reduce la fenomenicidad a dos tipos, ¿anula toda posibilidad de gradación? Y, por último, si la clasificación "metafísica" se reemplaza por una mirada hermenéutica, como propone Vinolo, ¿el fenómeno pierde la iniciativa? ${ }^{22}$

El punto de partida para responder estos interrogantes debe ser la definición heideggeriana de fenómeno que Marion sostiene siempre: fenómeno es lo que se da a partir de sí mismo. Este soi del fenómeno es lo que permite reconducir toda fenomenalización a su donación. Por este motivo, Marion entiende que todo fenómeno tiene un carácter acontecial. Esta cuestión se desarrolla en especial en su reciente Reprise du donné. La acontecialidad, señala el autor:

no define sólo un tipo entre otros de fenómenos (los acontecimientos de la historia, colectiva e individual), ni sólo a los fenómenos saturados (según la tópica del acontecimiento, el ídolo, la carne y el rostro del otro), sino que caracteriza esencialmente la propiedad de los fenómenos de aparecer siempre - cualquiera sea su diferencia de grado — por su propia iniciativa [...] y de sustraerse así a su aparente objetivación. (Marion 2016, p. 185)

Esto implica entonces que la donación de todo fenómeno se da en el modo de la saturación. En palabras de Marion:

La donación del fenómeno como acontecimiento (por lo tanto, la donación de todo fenómeno, en un grado u otro) incluye en ella su exceso sobre sí. La donación se cumple siempre en demasía. La saturación de ciertos fenómenos debe entenderse como la consecuencia formal de su fenomenicidad a la medida de la donación. Una donación sin demasía se contradiría. (Marion 2016 p. 186). ${ }^{23}$

${ }^{22}$ Claudia Serban se hace la misma pregunta: “¿Podemos aceptar que la mirada decida el acontecimiento y mantener el compromiso de Étant donné a favor del 'sí' del fenómeno, compromiso que estatuye que la 'iniciativa pertenece en principio al fenómeno y no a la mirada'?" (Serban 2012, p. 92).

${ }^{23}$ En La rigueur des choses, Marion ya anticipa esta afirmación: "Pienso cada vez más que, entre todos los fenómenos saturados, lo más determinante se encuentra en el acontecimiento; por lo tanto, pienso que todos los otros tipos de fenómenos

Revista de Filosofía Diánoia, vol. 65, no. 84 (mayo-octubre de 2020) e-ISSN: 1870-4913 • DOI: https://doi.org/10.22201/iifs.18704913e.2020.84.1586 
Aprehender un fenómeno en la medida de su donación implica aprehenderlo como fenómeno saturado; implica entregarse a la demasía, a la saturación que la donación del fenómeno desde sí conlleva. Por el contrario, la objetivación constituye un procedimiento inverso. Ya no se trata de entregarse a los términos en los que el fenómeno se da, comprometiendo nuestra subjetividad receptora según el punto de vista de la anamorfosis impuesta por lo dado. ${ }^{24}$ La objetivación constituye al objeto según los términos que impone el yo a la cosa y, de este modo, establece la primacía del "poder de conocer" del sujeto.

El objeto no se rige en realidad por la verdad de su existencia (ni de su esencia), sino (por la puesta en orden por nosotros en vista de producir evidencia para nosotros. [...] Más esencial para el objeto que la cosa, se encuentra por lo tanto en él o, más exactamente, fuera de él, el poder de conocer que lo constituye en cuanto que cognoscible y pensable (cogita$b l e$ ), el poder del Yo trascendental, aquel que ya está en el ego cogito tal como nos instaura a nosotros, los hombres, "como amos y poseedores de la naturaleza". (Marion 2016, p. 162)

Esta operación de objetivación pone entre paréntesis la cosa mediante la eliminación del componente variable, contingente, indeterminado de la hýle, y con la retención sólo de los elementos que satisfacen la única condición de la ciencia: la certeza (Marion 2016, pp. 154-156).

Por el contrario, en el caso del acontecimiento, se trata de exponer nuestra subjetividad de modo receptivo al "poder de aparecer" del fenómeno, adoptando el punto de vista que éste nos impone. De este modo, ya no es posible la supuesta observación neutral de la teoría y la ciencia, sino que es preciso involucrarnos:

[E]s preciso, no sólo que una mirada sea curiosa, disponible y ejercida, sino sobre todo que se someta a las exigencias de la figura que hay que ver. [...] [Se trata de] renunciar a organizar la visibilidad a partir de una decisión libre o del lugar propio de un espectador sin compromiso, para que la visibilidad se deje dictar por el fenómeno mismo, en [su] sí. (Marion 1998, p. 176)

saturados, cada uno a su manera, se encuentran regidos por la acontecialidad" (Marion 2012a, p. 270).

${ }^{24} \mathrm{El}$ autor se vale del dispositivo pictórico de la anamorfosis para explicar cómo lo dado impone el punto de vista desde el cual debe ser aprehendido ( $c f r$. Marion 1998, pp. 169-185).

Revista de Filosofía Diánoia, vol. 65, no. 84 (mayo-octubre de 2020)

e-ISSN: 1870-4913 • DOI: https://doi.org/10.22201/iifs.18704913e.2020.84.1586 
Se trata de dar lugar a la dimensión páthica receptiva en la que somos afectados por el fenómeno al recibirlo sin condiciones. Como bien recuerda Patricio Mena Malet, es por este rasgo de la recepción del acontecimiento que algunos filósofos de la generación de Marion, la llamada nouvelle phénoménologie (como Maldiney y Romano), han reflexionado sobre el dictum de Esquilo: tó pathei mathós, es preciso padecer o hacer la prueba para aprender (Mena Malet 2018, p. 242).

No obstante, ¿cuál es el sentido de enfatizar esta opción dicotómica? Marion es claro al respecto. Como respuesta en cierto modo a la objeción de Rogozinski, la contraposición entre el objeto y el acontecimiento devuelve la fuerza crítica a su propuesta filosófica. Como el ícono permitía advertir las limitaciones del ídolo en el campo teológico, el acontecimiento hace posible señalar el alcance del objeto en el campo fenomenológico. De este modo, la filosofía recupera su función decisiva. Si la filosofía se limita a trabajar con objetos, sin advertir la dimensión acontecial, entonces pierde su lugar propio. Si la filosofía olvida que existe una decisión filosófica previa a la objetivación que alcanzan las ciencias exactas, entonces - según Marion- comete un doble error. En primer lugar, el de propiciar su propia marginación al devenir una mera comentarista tardía y "frecuentemente superficial o anecdótica" de la práctica de las ciencias exactas, perdiendo su autoridad y su campo propio. En segundo lugar, el error de privilegiar el modelo matemático que desmaterializa (desde Platón, pasando por Descartes y hasta Husserl y Carnap) y conduce a la reducción del saber a las ciencias del objeto (Marion 2016, pp. 164-165).

Se trata, pues, de recuperar para la filosofía, ya no su lugar de "ciencia rigurosa" o de "filosofía primera", pero si su papel crítico de "filosofía última" (cfr. Marion 2001, pp. 1-34), capaz de actuar como el "correctivo fenomenológico" o como el "correctivo acontecial" de la mirada objetivadora, en los casos en que éste se requiera. ${ }^{25}$

En este sentido, aunque considero en buena medida acertadas las críticas de Steinbock y de Gschwandtner respecto de la necesidad de introducir una gradación, entiendo que no hay que perder de vista el poder crítico de la propuesta de Marion. Si bien los últimos textos del

${ }^{25}$ En una lectura del joven Heidegger, Marion sostiene: "El Ereignis se constituirá así como la corrección fenomenológica que, de un extremo al otro, asegura a los ojos de Heidegger el acceso fenomenológico (y no teórico) al es gibt. Será entonces esta corrección la que deberá discutirse en el momento en que se trate de medir hasta dónde llegó la fenomenología de la donación y a partir de qué punto está precluida" (Marion 2012b, p. 58). Para una lectura detenida de la función crítica de la fenomenología de Marion como “filosofía última”, véase Roggero 2018.

Revista de Filosofía Diánoia, vol. 65, no. 84 (mayo-octubre de 2020) e-ISSN: 1870-4913 • DOI: https://doi.org/10.22201/iifs.18704913e.2020.84.1586 
pensador francés parecen enfatizar cada vez más esta opción dicotómica, la idea y la necesidad de una gradación, aunque pierde protagonismo, no desaparece de su consideración: la objetivación admite grados y la acontecialidad también. Sin duda, se trata de un aspecto - como bien señala Gschwandtner- poco desarrollado por el propio Marion pero no implica contradecir la lógica de su propuesta, sino que puede complementarla (cfr. Gschwandtner 2014, p. 24).

No obstante, cabe señalar una cuestión importante. Gschwandtner y el propio Marion en más de un pasaje hablan de "grados de donación" (cfr. Gschwandtner 2014, pp. 5, 44, 52, 80, 84, 125, 139-140, 144, 170, 181-183, 192-193, 196, 201-203 y Marion 1998, pp. 45, 310, 325, 420). Según lo que manifiesta en otros pasajes posteriores de su obra y que, por lo tanto, pueden entenderse en parte como correcciones, Marion enfatiza que la donación siempre se da en demasía, en exceso, con carácter saturante (cfr. Marion 2016, p. 186). En este sentido, es posible afirmar que todo fenómeno es, al menos en potencia, un fenómeno saturado. Por lo tanto, lo que es graduable es la fenomenicidad, no la donación (cfr. Marion 2012a, p. 179, y Marion 2016, p. 179), pues en esta cuestión es en la que tiene injerencia la subjetividad receptora: si el yo interviene activamente tratando de controlar el exceso de lo dado, la indeterminación de la hýle de la cosa, se registrará una gradación de objetivación que puede trazarse desde el fenómeno pobre al fenómeno común e introducir las variantes que se consideren necesarias. A la inversa, cuando el yo abandona su posición constituyente y se entrega a los dictados y exigencias del modo de darse de la cosa, estaremos frente a distintos grados de saturación que irán desde los fenómenos saturados en primer grado hasta los fenómenos de revelación.

Si la fenomenología debe alcanzar su cometido último, que no es otro que acceder a las cosas mismas, tiene que adoptar la radicalidad capaz de suspender el "poder de conocer" del sujeto en favor del "poder de aparecer" del fenómeno. Tal es la vía que la fenomenología heideggeriana anuncia desde su concepto de fenómeno como automostración y que la fenomenología de Marion desarrolla. Pero, ¿cómo puede ser posible que el fenómeno tome la iniciativa? ¿Qué significa que algo "se muestre"? ¿Qué significa que haya un soi del fenómeno? La indagación de este "sí" del fenómeno lleva a Marion a distinguir la instancia de la fenomenalización de la instancia de la donación, que Heidegger no distingue con claridad. La tesis de Étant donné es precisamente que "todo lo que se muestra se da" (Marion 1998, p. 424). La acontecialidad del fenómeno radica en que el "sí" de la fenomenalización es el "sí" de la donación. Ésa es la tesis del capítulo de De surcroît sobre el aconteci- 
miento (cfr. Marion 2001, pp. 35-63). Todo fenómeno saturado del tipo del acontecimiento adviene desde y por sí mismo sin que sea posible establecer cuál es su causa u origen. Pero este rasgo acontecial, que en principio sólo parece concernir al acontecimiento en sentido estricto, se extiende a todos los fenómenos con la nueva tópica de los fenómenos. El fenómeno saturado, categoría fundamental de la fenomenología de Marion, es el paradigma de toda fenomenicidad, pues todo fenómeno se da como fenómeno saturado en la medida en que se da a partir de sí mismo.

\section{Referencias bibliográficas}

Falque, Emmanuel, 2004, Métamorphose de la finitude. Essai philosophique sur la naissance et la résurrection, Cerf, París.

Falque, Emmanuel, 2014, "Phénoménologie de l'extraordinaire (J.-L. Marion)", en Emmanuel Falque, Le combat amoureux. Disputes phénoménologiques et théologiques, Hermann, París, pp. 137-193 [1a. ed.: Philosophie, vol. 3, no. 78, 2003, pp. 52-76].

González di Pierro, Eduardo, 2011, "Gegebenheit y donation: dos modos de la dación fenomenológica. La crítica de Marion a Husserl. Coincidencias y divergencias", Devenires, vol. 12, no. 23, pp. 123-133.

Gschwandtner, Christina M., 2014, Degrees of Givenness. On Saturation in JeanLuc Marion, Fordham University Press, Nueva York.

Heidegger, Martin, 1977, Gesamtausgabe I. Abteilung: Veröffentlichte Schriften 1914-1970. Band 2. Sein und Zeit, Vittorio Klostermann, Fráncfort del Meno.

Heidegger, Martin, 1987, "Die Idee der Philosophie und das Weltanschauungsproblem", en Martin Heidegger, Gesamtausgabe II. Abteilung: Vorlesungen. Band 56/57. Zur Bestimmung der Philosophie, Vittorio Klostermann, Fráncfort del Meno, pp. 1-62.

Heidegger, Martin, 2007, "Das Ende der Philosophie und die Aufgabe des Denkens", en Martin Heidegger, Gesamtausgabe I. Abteilung: Veröffentlichte Schriften 1914-1970. Band 14. Zur Sache des Denkens, Vittorio Klostermann, Fráncfort del Meno, pp. 67-90.

Husserl, Edmund, 1973, Husserliana. Gesammelte Werke. Band I. Cartesianische Meditationen und Pariser Vorträge, Martinus Nijhoff, La Haya.

Husserl, Edmund, 1976, Husserliana. Gesammelte Werke. Band III/1. Ideen zur einer reiner Phänomenologie und phänomenologischen Philosophie. Erstes Buch. Text der 1.-3. Auflage, Martinus Nijhoff, La Haya.

Husserl, Edmund, 1984, Husserliana. Gesammelte Werke. Band XIX/1. Logische Untersuchungen. Zweiter Band. Erster Teil. Untersuchugen zur Phänomenologie und Theorie der Erkenntnis, Martinus Nijhoff, La Haya.

Husserl, Edmund, 1987, Husserliana. Gesammelte Werke. Band XXV. Aufsätze und Vorträge (1911-1921), Martinus Nijhoff, La Haya.

Revista de Filosofía Diánoia, vol. 65, no. 84 (mayo-octubre de 2020) e-ISSN: 1870-4913 • DOI: https://doi.org/10.22201/iifs.18704913e.2020.84.1586 
Janicaud, Dominique, 2009, "La phénoménologie éclatée", en Dominique Janicaud, La phénoménologie dans tous ses états, Gallimard, París, pp. 151-276.

Kant, Immanuel, 1903-1904, Kritik der reinen Vernunft, Georg Reimer (Akademie Ausgabe, III y IV), De Gruyter, Berlín.

Lavigne, Jean-François, 2003, Husserl et la naissance de la phénoménologie. 1909-1913, Presses Universitaires de France, París.

Marion, Jean-Luc, 1989, Réduction et donation. Recherches sur Husserl, Heidegger et la phénoménologie, Presses Universitaires de France, París.

Marion, Jean-Luc, 1992, "Le phénomène saturé", en Jean-Luc Marion, Michel Henry, Jean-Louis Chrétien y Paul Ricœur, Phénoménologie et théologie, Criterion, París, pp. 79-128.

Marion, Jean-Luc, 1998, Étant donné. Essai d’une phénoménologie de la donation, 2a. ed. corregida, Presses Universitaires de France, París.

Marion, Jean-Luc, 2001, De surcroît. Études sur les phénomènes saturés, Presses Universitaires de France, París.

Marion, Jean-Luc, 2005, Le visible et le révélé, Cerf, París.

Marion, Jean-Luc, 2010, Certitudes négatives, Grasset, París.

Marion, Jean-Luc, 2012a, La rigueur des choses. Entretiens avec Dan Arbib, Flammarion, París.

Marion, Jean-Luc, 2012b, Figures de phénoménologie. Husserl, Heidegger, Lévinas, Henry, Derrida, Vrin, París.

Marion, Jean-Luc, 2016, Reprise du donné, Presses Universitaires de France, París.

Mena Malet, Patricio, 2018, "Pathos y phainomenon. El acontecimiento como fenómeno insigne y la aventura de su recepción", en Felipe Johnson, Patricio Mena y Samuel Herrera (comps.), ¿Hacia las cosas mismas? Discusiones en torno a la problemática claridad del fenómeno, Ediciones Universidad de la Frontera, Temuco, pp. 217-255.

Pradelle, Dominique, 2012, Par-delà de la révolution copernicienne. Sujet transcendantal et facultés chez Kant et Husserl, Presses Universitaires de France, París.

Ricœur, Paul, 1950, "Note 1, p. 78", en Edmund Husserl, Idées directrices pour une phénoménologie et une philosophie phénoménologique pures, trad. y notas Paul Ricœur, Gallimard, París.

Roggero, Jorge Luis, 2018, "La 'filosofía última' de J.-L. Marion", Tópicos. Revista de Filosofía, núm. 55, pp. 31-60, <https://doi.org/10.21555/top.v0i55. 936>.

Rogozinski, Jacob, 2015, “Questions à J.-L. Marion”, en Jean-Luc Marion, Ce que nous voyons et ce qui apparaît, Ina, París, pp. 74-77.

Serban, Claudia, 2012, "La méthode phénoménologique, entre réduction et herméneutique", Les Études Philosophiques, vol. 100, no. 1, pp. 81-100, $<$ https://doi.org/10.3917/leph.121.0081>.

Steinbock, Anthony J., 2007, "The Poor Phenomenon: Marion and the Problem of Givenness", Alter. Revue de Phénoménologie, vol. 15, pp. 357-372.

Revista de Filosofía Diánoia, vol. 65, no. 84 (mayo-octubre de 2020)

e-ISSN: 1870-4913 • DOI: https://doi.org/10.22201/iifs.18704913e.2020.84.1586 
Vinolo, Stéphane, 2017, "La tentation moderne de Jean-Luc Marion: le scandale de la saturation", Dialogue. Revue Canadienne de Philosophie, vol. 55, no. 2, pp. 343-362, <https://doi.org/10.1017/S0012217316000469>.

Recibido el 21 de noviembre de 2018; revisado el 12 de junio de 2019; aceptado el 13 de junio de 2019. 